\title{
RECENT WORK ON KERR STABILITY AND \\ SUPERRADIANT WAVE SCATTERING
}

\author{
WILLIAM H. PRESS
}

California Institute of Technology, Pasadena, Calif., U.S.A.

\begin{abstract}
As a stopgap measure until a rigorous analytic determination is made, Teukolsky and I have tested the dynamical stability of the Kerr metric under small perturbations numerically (Press and Teukolsky, 1973). We find that it is stable for all $a \leqslant M$. We have also computed the magnitude of electromagnetic and gravitationalwave amplification in superradiant scattering, and Bardeen has independently obtained identical results (Teukolsky, 1973; Teukolsky et al., 1974). The amplification ranges up to $\sim 2 \%$ for electromagnetism $(l=m=1)$ and up to $140 \%$ for gravitation $(l=m=2)$; these values are also consistent with Starobinsky's (1973) results for the value of critical frequencies.
\end{abstract}

\section{References}

Press, W. H. and Teukolsky, S. A.: 1973, Astrophys. J. 185, 649.

Starobinsky, A. A.: 1973, Zh.E.T.F. 65, 3.

Teukolsky, S. A.: 1973, unpublished Ph.D. Thesis, available from University Microfilms, Inc., Ann Arbor, Michigan.

Teukolsky, S. A., Bardeen, J. M., and Press, W.: 1974, in preparation. 